\title{
Quantitative Low-Voltage STEM imaging in the Presence of Temporal Incoherence
}

Mark P. Oxley ${ }^{1,2}$ and Stephen J. Pennycook ${ }^{2,1}$

${ }^{1 .}$ Department of Physics and Astronomy, Vanderbilt University, Nashville, Tennessee, USA

2. Materials Science and Technology Division, Oak Ridge National Laboratory, Oak Ridge, Tennessee, USA

In the STEM spatial incoherence, caused by a finite electron source size, can be included in image simulation by convolution with a suitable function describing the source distribution. This has successfully been applied to atomic resolution STEM imaging at $300 \mathrm{kV}$ to provide quantitative agreement between simulation and experiment for a range of different experimental conditions [1]. At these high energies, temporal incoherence, related to the variation in incident electron energy, can largely be ignored. Many materials however require the use of lower incident energies to avoid specimen damage [2,3]. At low energies, such as $60 \mathrm{kV}$ or lower, an accurate description of temporal incoherence is essential to achieve quantitative agreement between theory and experiment.

Unlike spatial incoherence, which is usually determined by applying a Gaussian blur to simulated images in order to match experimental image contrast [1], the energy spread $\Delta E$ of a given machine can be determined precisely using an electron energy loss spectrometer. Using the known chromatic aberration $C_{\mathrm{c}}$ of the imaging system, this energy spread may then be converted into a defocus spread $\Delta f=C_{\mathrm{c}} \Delta E / E$, where $E$ is the incident electron energy. For fixed energy spread and $C_{\mathrm{c}}$ the defocus spread is inversely proportional to incident energy.

Shown in Fig. 1 is the energy spread for a Gaussian distribution with a FWHM of $0.35 \mathrm{eV}$ and the measured energy spread of ORNL's Nion UltraSTEM. The cold FEG source does not provide a symmetric energy spread and is poorly described by a Gaussian. Figure 1 also shows the effects of these distributions on the probe intensity for incident energies of both 40 and $60 \mathrm{kV}$. Unlike spatial incoherence the effect of temporal incoherence is not a simple blur. Instead the peak intensity is reduced as intensity is moved into the probe tails. Earlier work has sought to remove these tails via a Fourier filter [2], but here we include a full description of temporal incoherence in the image simulations.

Shown in Fig. 2 are $60 \mathrm{Kv}$ ADF image simulations of graphene for no energy spread and each of the energy spreads shown in Fig. 1. A Gaussian blur of $0.75 \AA$ has been applied to account for source size effects. At first glance, there seems little difference between the Gaussian energy spread and the measured energy spread. Both lead to a reduction in contrast but the effect is subtlety different from a simple Gaussian blur applied after the image is calculated. The intensity centre of the $\mathrm{C}_{6}$ ring is increased, and the peak heights are reduced, but the intensity between the dumbbells remains approximately constant.

While the Gaussian spread and the measured energy spread provide qualitative agreement for ADF imaging of perfect graphene, it is the identification of impurities in these 2D structures that is of broad general interest $[2,3]$ which is achieved assuming that the peak STEM ADF intensity is described by $I \propto Z^{n}$ where $n=2$ corresponds to back scattering and is reduced for ADF. Figure 2 also shows the variation in peak intensity for a number of impurities placed in the graphene ring. There is a strong variation of the fitted value of $n$ depending on the description of temporal incoherence. The measured 
energy spread results in an intensity for $\mathrm{Si}$ which would correspond to $\mathrm{Mg}$ if no temporal incoherence was assumed.

Differences in peak intensities are even more pronounced at lower incident energies. We will discuss the importance of accurate determination of both temporal and spatial incoherence parameters of the microscope, and the use of detailed image simulation in order to provide quantitative analysis of the resulting images.

References:

[1] JM LeBeau et al, Phys. Rev. Lett. 100 (2008) 206101

[2] OL Krivanek et al, Nature 464 (2011), p. 571.

[3] W Zhou et al, Microsc. Microanal. 18 (2012), p. 1342.

[4] This work was supported in part by DOE Grant No. DE-FG02-09R46554 (M.P.O.) and by the DOE Office of Basic Energy Sciences, Materials Sciences and Engineering Division (S.J.P.).
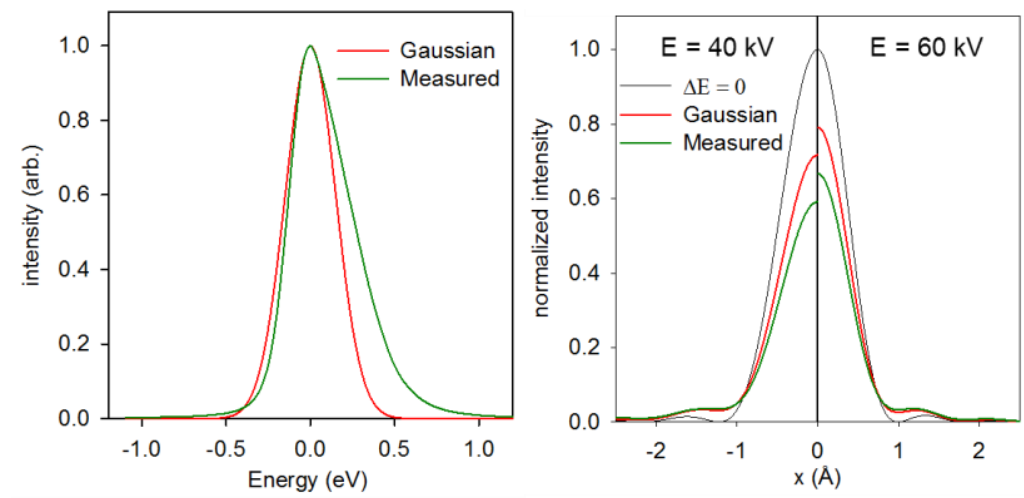

Figure 1. Left: Energy spread assuming a $0.35 \mathrm{eV} \mathrm{FWHM} \mathrm{Gaussian} \mathrm{(red)} \mathrm{compared} \mathrm{to} \mathrm{the} \mathrm{measured}$ (green) energy spread. Right: Probe intensities for 40 and $60 \mathrm{kV}$ incident energies with probe forming apertures of 30 mrads. Intensities are shown for no energy spread (black), a Gaussian energy spread (red) and the measured energy spread (green).
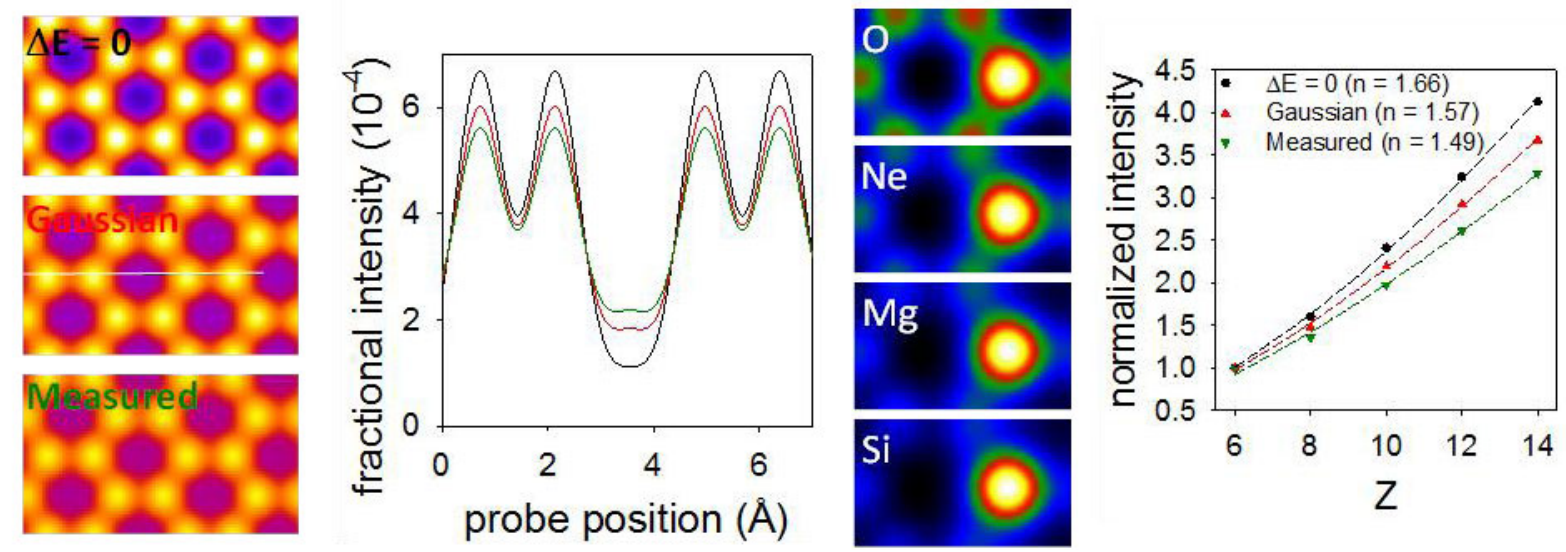

Figure 2. Left: Simulated STEM ADF of images graphene for each of the $60 \mathrm{kV}$ probes shown above. Right: Simulated STEM ADF images for a range of impurities in graphene and the corresponding peak intensities as a function of atomic number $\mathrm{Z}$. 